\title{
СОВРЕМЕННОЕ СОСТОЯНИЕ И ПЕРСПЕКТИВЫ ЛЕЧЕНИЯ КОЛОРЕКТАЛЬНЫХ ОПУХОЛЕЙ ПРИ УДАЛЕНИИ ОБРАЗЦОВ ИЗ ЕСТЕСТВЕННЫХ ОТВЕРСТИЙ (N.O.S.E.S.)
}

\begin{abstract}
(
CURRENT STATE AND PROSPECTS FOR TREATMENT OF COLORECTAL TUMORS WHEN EXTRACTION OF SAMPLES FROM NATURAL HOUSINGS (N.O.S.E.S.)

$\mathrm{CaO} \mathrm{Yu}$

Zhang Xinliang

Fu Yu

Summary. Colorectal cancer (CRC) is one of the most common malignant tumors of the digestive tract. Recently, there has been an increase in overall morbidity and mortality from CRC, while surgery remains the most effective treatment for CRC. Thanks to the constant modernization of surgical and diagnostic technologies, the constantly improving minimally invasive surgical technique has become one of the standards in the treatment of colorectal cancer. As much attention is paid to the patient's quality of life after surgery as to the oncological and surgical outcome. Thus, both endoscopic transluminal surgery (N.O.T.S.E.) and natural orifice surgery (N.O.S.E.S.) are widely used today, essentially displacing open methods of treatment. In this work, we consider the main methods and historical aspects of the development of N.O.S.E.S. in the treatment of colorectal tumors. It was revealed that N.O.S.E.S. in the treatment of colorectal tumors requires improved extraction methods to prevent local recurrence and infectious complications, for the purpose of which it is necessary to use high-precision endoscopic auxiliary instruments, robotic surgery, and to improve sterilization and washing protocols of the extraction pathways.
\end{abstract}

Keywords: N.O.S.E.S., N.O.T.S.E., colorectal cancer, laparoscopic surgery, malignant tumors.

\author{
Цао Юй \\ Аспирант, Первый Московский государственный \\ медицинский университет имени И.М. Сеченов \\ goodmancaoyu@gmail.com \\ Чжан Синьлян \\ Аспирант, Первый Московский государственный \\ медицинский университет имени И.М. Сеченова \\ zx10620@yandex.ru \\ Фу Юŭ \\ Первый Московский государственный медицинский \\ университет имени И.М. Сеченов \\ a840534855@gmail.com
}

Аннотация. Колоректальный рак (КРК) — одна из наиболее распространенных злокачественных опухолей пищеварительного тракта. В последнее время наблюдается повышение общей заболеваемости и смертности от КРК, при этом хирургическое вмешательство остается наиболее эффективным способом лечения КРК. Благодаря постоянной модернизации хирургических и диагностических технологий, постоянно совершенствующаяся минимально инвазивная хирургическая техника стала одним из стандартов в лечении колоректального рака. Качеству жизни пациента после операции уделяется столько же внимания, сколько и онкологическому и хирургическому результату. Таким образом, как эндоскопическая транслюминальная хирургия (N.0.T.S.E.), так и хирургия через естественные отверстия (N.O.S.E.S.) на сегодняшний день широко применяются, существуенно сместив открытые методы лечения. В данной работы мы рассматриваем основные методы и исторические аспекты развития хирургии N.O.S.E.S. при лечении колоректальных опухолей. Было выявлено, что хирургия N.O.S.E.S. в лечении колоректальных опухолей требует усовершенствования методов экстракции для предотвращения локальных рецидивов и инфекционных осложнений, с целью которых необходимо применять высокоточные эндоскопический вспомогательные инструменты, роботическую хирургию и улучшать стерилизацию и промывные протоколы путей экстракции.

Ключевые слова: N.O.S.E.S., N.O.T.S.E., колоректальный рак, лапароскопическая хирургия, злокачественные опухоли.

ческой хирургии (N.O.T.E.S.) [2]. Данный вид эндоскопии проводится через естественные отверстия рта, влагалища, уретры, ануса и т.д. для диагностики или лечения заболевания [3]. В лечении колоректальных опухолей, активное развитие получил метод N.O.S.E.S. N.O.S.E.S объединяет концепцию N.O.T.E.S и преимущества традиционной лапароскопической хирургии [4]. В настоящее время в клинической литературе имеется множество 
сообщений о клиническом применении метода N.O.S.E.S. и его модификаций. С целью систематизации данных о современных аспектах N.O.S.E.S проведен обзор существующих публикаций в области классификации, использования и вариаций N.O.S.E.S.

\section{К^ассификашия}

В настоящее время метод N.O.S.E.S. используется для лечения патологий внутренних органов, наиболее часто при лечении колоректальных новообразования. Для доброкачественных и злокачественных опухолей прямой кишки используются 2 основных типа в зависимости от способа получения образцов: трансвагинальный и трансанальный N.O.S.E.S.

\section{Трансвагинальный N.O.S.E.S}

Благодаря хорошей пластичности влагалища, обильному кровоснабжению и быстрому заживлению ран, трансвагинальный N.O.S.E.S. подходит для пациенток, у которых крупный размер опухоли, которую невозможно извлечь через трансанальный доступ. В 2015 году был описан метод комбинированного роботизированного радикального иссечения рака желудка с трансвагинальным N.O.S.E.S. с реконструкцией пищеварительного тракта ручным наложением шва под оптическим микроскопом [5]. Послеоперационный период при применении данного метода протекал без осложнений, причем средняя продолжительность пребывания в больнице составила всего 3,6 дня. В ходе другого исследования выполнялось трансвагинальное N.O.S.E.S. по заднему своду влагалища, который является наиболее растяжимой частью и наиболее легко идентифицируемым анатомическим ориентиром при лапароскопии, а положение заднего свода влагалища глубокое и вокруг него нет активного нервного и сосудистого сплетения, поэтому травма в этом месте не окажет очевидного влияния на сексуальную жизнь пациента [6].

Трансвагинальный N.O.S.E.S. имеет недостатки, поскольку при данной процедуре дополнительно повреждаются внутренние органы, а анатомия ректовагинальной области сложна и изменчива, что значительно усложняет применение данного доступа. Поэтому не рекомендуется применять трансвагинальный N.O.S.E.S. женщинам с острой гинекологической инфекцией, аномалиями влагалища или женщинам, не состоящим в браке и планирующим беременность.

\section{Трансанальный N.O.S.E.S.}

Трансанальный N.O.S.E.S. подходит для пациентов с небольшими новообразованиями, и не имеет ограничений по полу [7]. В 1993 году Дж. Франклин с соавтора- ми впервые провели операцию лапароскопической резекции сигмовидной кишки с трансанальной N.O.S.E.S., средняя продолжительность послеоперационного пребывания в больнице ч составила всего 3.4 дня [8]. В ходе исследований было выявлено, что модифицированная техника лапароскопической передней резекции с трансанальной N.O.S.E.S. соответствует принципу безконтактной техники опухолевой экстракции и имеет такие преимущества, как меньшая травматизация, быстрое восстановление и меньшее количество осложнений [9]. Также было выявлено, что трансанальный N.O.S.E.S. не имеет ограничений по полу пациента, поэтому в настоящее время он применяется наиболее широко [10].

Сегодня принято делить N.O.S.E.S. на три категории:

а) Выльгусный тип резекции. Показания в основном для низких резекций прямой кишки.

b) Тип резекция-удаления. Применимо для срединных резекций прямой кишки.

с) Тракционный тип резекции. Адаптирован и применим в лечении ректальной, сигмовидной, левой и правой ободочной кишки, а также при полной колэктомии.

N.O.S.E.S.1 также разделяют на подтипы: А (метод выворота), В (метод модифицированного выворота), С (колоанальный анастомоз, метод Паркса), D (резекция межсфинктерного пространства, метод ISR), E (извлечение трансанального канала толстой кишки, метод Bacon), F (предложен Петров В. Царьковым) [11]

\section{Преимушества}

В 2011 году был опубликован систематический обзор, включивший 94 случаев. Было выявлено, что гемиколэктомии слева процедурой N.O.S.E.S. является безопасной и выполнимой [12]. На данный момент не обнаружено значительных различий во времени до появления первых газов, кровотечения, непроходимости, запора, и инфекционных осложнений между традиционным лапароскопическим и лапароскопическим трансанальным извлечением образца N.O.S.E.S., но значительные различия в частоте послеоперационной диареи, уровне боли и удовлетворенности эстетикой брюшной стенки в пользу N.O.S.E.S.

Чжан Синмао и др. сравнили N.O.S.E.S., выполненный с помощью традиционной лапароскопии и тотальной лапароскопии, и обнаружил, что полная лапароскопическая резекция N.O.S.E.S. имеет несколько преимуществ по сравнению с лапароскопической ассистированной резекцией. Было выявлено, что колоректальное N.O.S.E.S.:

- Оставляет несколько небольших рубцов на брюшной стенке. 
- Эффективно уменьшая послеоперационную боль у пациентов.

- Снижает риск послеоперационной грыжи и осложнений, связанных с инфекцией.

- Способствует раннему послеоперационному передвижению пациентов, откашливанию мокроты.

- Снижает возникновение многих осложнений, таких как послеоперационная кишечная непроходимость, венозный тромбоз нижних конечностей и легочная инфекция [14].

Преимущества N.O.S.E.S очевидны: хороший косметический результат, отсутствие статистической разницы по эффективности лечения и длины безрецидивного периода, эффективности онкологического контроля.

\section{Осложнения}

Существенное различие между N.O.S.E.S. и традиционной лапароскопической хирургии - путь экстракции опухолевой ткани. N.O.S.E.S. позволяет избежать дополнительной травматизации брюшной стенки и связанных с ним осложнений, однако N.O.S.E.S. имеет потенциальные риски распространения клеток опухоли, диссеминации клеток в область брюшной полости на пути экстракции. Масаюки с соавторами рекомендуют применять солевой раствор, содержащий иодофоры для неоднократного промывания путей экстракции, а также выполнять цитологическое обследование промывочного раствора интраоперационно, чтобы подтвердить отсутствие раковых клеток [15].

Гюндоган и др. описал случай имплантации опухолевых клеток у пациентки, перенесшей трансвагинальное удаление образцов при левой колэктомии. У пациентки развилась аденокарцинома в вагинальной стенке спустя 1 год после операции. Как результат, матка, фаллопиевы трубы, задняя стенка влагалища были удалены при повторном вмешательстве [16].

При выполнении колоректального N.O.S.E.S. увеличивается возможность развития послеоперационных инфекционные осложнений в связи с выведением кишечника через стенку брюшной полости. В 2011 году Leroy et al. сообщили о 16 случаях N.O.S.E.S. сигмовидного отдела толстой кишки при которых потребовалась интерпозиаиця кишечника [17].

В 2016 году группа ученых продемонстрировала, что на ранних этапах бактериологического обсеменения при выполнении колоректального N.O.S.E.S. не определялось послеоперационного сепсиса или раневой инфекции, не было обнаружено опухолевых клеток в экссудате у пациентов при обработке путей экстракции большим количеством раствора для стерилизации кишечной полости [18].

Многие современные ученые считают, что лечение колоректального рака с помощью трансанального N.O.S.E.S. является безопасным методом с превосходящими косметическими результатами.

При выполнении колоректальных N.O.S.E.S., если опухоль крупных размеров, это может привести к разрыву или повреждению стенки прямой кишки во время экстракции образца. В 2013 году, Uccella и других [19] проанализировали 230 пациенток с трансвагинальным N.O.S.E.S. с целью определения частоты промежностных осложнений. Авторы показали, что трансвагинальных N.O.S.E.S. не оказывает прямое негативное влияние на половую функцию и удерживающую функцию промежностных мышц.

Исследование, проведенное группой ученых под руководством Сухраба Saurabh показало, что трансвагинальный N.O.S.E.S. может привести к нарушению половой функции и даже к бесплодию. В 2015 году проспективное исследование [8] показало, что по сравнению с обычной лапароскопической колоректальной хирургией, при выполнении трансанального N.O.S.E.S. ни у одного пациента не было тяжелой анальной дисфункции через 6 и 12 недель после операции. В 2016 году Нгу и его коллеги выявили, показали, что после трансанального N.O.S.E.S. не было выявлено случае недержания кала при среднем периоде наблюдения 9 месяцев после операции.

\section{Зак^ючение}

Современные данные мировой литературы единогласно утверждают, что N.O.S.E.S. для лечения колоректальных опухолей - безопасная и эффективная процедура с превосходящими косметическими результатами. По сравнению с традиционной лапароскопической хирургией, N.O.S.E.S. характеризуется более высоким уровнем послеоперационного качества жизни, лучшими косметическими результатами, улучшенными результатами психосоциальной реабилитации, результатами онкологического лечения и без рецидивного периода. Безусловно, хирургия N.O.S.E.S. в лечении колоректальных опухолей требует усовершенствования методов экстракции для предотвращения локальных рецидивов и инфекционных осложнений, с целью которых необходимо применять высокоточные эндоскопический вспомогательные инструменты, роботическую хирургию и улучшать стерилизацию и промывные протоколы путей экстракции. Несмотря на существующие недостатки, минимально- инвазивная методика N.O.S.E.S. набирает популярность и находит большее применение в клинической практике ежегодно. 


\section{ЛИТЕРАТУРА}

1. Leung AL, Cheung HY, Li MK. Advances in laparoscopic colorectal surgery: a review on NOTES and transanal extraction of specimen [J]. Asian journal of endoscopic surgery, 2014, 7(1): 11-16.

2. Kalloo AN, Singh VK, Jagannath SB, et al. Flexible transgastric peritoneoscopy: a novel approach to diagnostic and therapeutic interventions in the peritoneal cavity [J]. Gastrointest Endosc,2004, 60(1): 114-117.

3. Zeng WG, Zhou ZX. Mini-invasive surgery for colorectal cancer [J]. Chinese journal of cancer, 2014, 33(6): 277-284.

4. Xu S, Liu K, Chen X, Yao H. The safety and efficacy of laparoscopic surgery versus laparoscopic NOSE for sigmoid and rectal cancer. Surg Endosc. 2021 Jan 21. doi: 10.1007/s00464-020-08260-6. Epub ahead of print. PMID: 33475847.

5. Zhang S, Jiang ZW, Wang G, et al. Robotic gastrectomy with transvaginal specimen extraction for female gastric cancer patients [J]. World J Gastroenterol, 2015, 21(47): 13332-13338.

6. Li XW, Wang CY, Zhang JJ, Ge Z, Lin XH, Hu JH. Short-term efficacy of transvaginal specimen extraction for right colon cancer based on propensity score matching: A retrospective cohort study. Int J Surg. 2019 Dec;72:102-108.

7. Nishimura A, Kawahara M, Honda K, Ootani T, Kakuta T, Kitami C, Makino S, Kawachi Y, Nikkuni K. Totally laparoscopic anterior resection with transvaginal assistance and transvaginal specimen extraction: a technique for natural orifice surgery combined with reduced-port surgery. Surg Endosc. 2013 Dec;27(12):4734-40.

8. Franklin ME Jr, Ramos R, Rosenthal D, Schuessler W. Laparoscopic colonic procedures [J]. World J Surg, 1993, 17(1): 51-56.

9. Yu S, Ji Y, Luo T, Xu P, Zhen Z, Deng J. A modified technique of transanal specimen extraction in the laparoscopic anterior rectal resection for upper rectal or lower sigmoid colon cancer: a retrospective study. BMC Surg. 2021 Feb 12;21(1):82.

10. Efetov SK, Tulina IA, Kim VD, et al. Natural orifice specimen extraction (NOSE) surgery with rectal eversion and total extra abdominal resection [J]. Tech Coloproctol, 2019, 23(9): 899-902.

11. Guan X, Liu Z, Longo A, Cai JC, Tzu-Liang Chen et al. International consensus on natural orifice specimen extraction surgery (NOSES) for colorectal cancer. Gastroenterol Rep (0xf). 2019 Feb;7(1):24-31.

12. Wei Chang Wai Ke Za Zhi. 2016 Jun;19(6):621-3. Chinese. PMID: 27353095.

13. Wolthuis AM, Van Geluwe B, Fieuws S, et al. Laparoscopic sigmoid resection with transrectal specimen extraction: a systematic review [J]. Colorectal disease: the official journal of the Association of Coloproctology of Great Britain and Ireland, 2012, 14(10): 1183-1188.

14. Xingmao Z, Haitao Z, Jianwei L et al. Totally laparoscopic resection with natural orifice specimen extraction (NOSE) has more advantages comparing with laparoscopicassisted resection for selected patients with sigmoid colon or rectal cancer. Int J Colorectal Dis. 2014 Sep;29(9):1119-24.

15. $\mathrm{Ng} \mathrm{HI}$, Sun WQ, Zhao XM, et al. Outcomes of trans-anal natural orifice specimen extraction combined with laparoscopic anterior resection for sigmoid and rectal carcinoma: An observational study [J]. Medicine (Baltimore), 2018, 97(38): e12347

16. Leroy J, Costantino F, Cahill RA, et al. Laparoscopic resection with transanal specimen extraction for sigmoid diverticulitis [J]. Br J Surg, 2011, 98(9): $1327-1334$.

17. Saurabh B, Chang SC, Ke TW, et al. Natural orifice specimen extraction with single stapling colorectal anastomosis for laparoscopic anterior resection: feasibility, outcomes, and technical considerations [J]. Dis Colon Rectum, 2017, 60(1): 43-50.

(с) Цао Юй ( goodmancaoyu@gmail.com ),

Чжан Синьлян ( zx10620@yandex.ru ),Фу Юй ( a840534855@gmail.com ).

Журнал «Современная наука: актуальные проблемы теории и практики» 